\title{
Propofol administration to the fetal-maternal unit reduces cardiac injury in late-preterm lambs subjected to severe prenatal asphyxia and cardiac arrest
}

\author{
Matthias Seehase ${ }^{1-3}$, Patrick Houthuizen ${ }^{4}$, Reint K. Jellema ${ }^{1,2}$, Jennifer J.P. Collins ${ }^{1}$, Otto Bekers ${ }^{5}$, Johannes Breuer ${ }^{3}$ and \\ Boris W. Kramer ${ }^{1,2}$
}

BACKGROUND: Cardiac dysfunction is reported to occur after severe perinatal asphyxia. We hypothesized that anesthesia of the mother with propofol during emergency cesarean section (c-section) would result in less cardiac injury (troponin T) in preterm fetuses exposed to global severe asphyxia in utero than anesthesia with isoflurane. We tested whether propofol decreases the activity of proapoptotic caspase-3 by activating the antiapoptotic AKT kinase family and the signal transducer and activator of transcription-3 (STAT-3).

METHODS: Pregnant ewes were randomized to receive either propofol or isoflurane anesthesia. A total of 44 late-preterm lambs were subjected to in utero umbilical cord occlusion (UCO), resulting in asphyxia and cardiac arrest, or sham treatment. After emergency c-section, each fetus was resuscitated, mechanically ventilated, and supported under anesthesia for $8 \mathrm{~h}$ using the same anesthetic as the one received by its mother.

RESULTS: At $8 \mathrm{~h}$ after UCO, the fetuses whose mothers had received propofol anesthesia had lower plasma troponin $T$ levels, and showed a trend toward a higher median left ventricular ejection fraction (LVEF) of 84\% as compared with 74\% for those whose mothers had received isoflurane. Postasphyxia activation of caspase-3 was lower in association with propofol anesthesia than with isoflurane. Postasphyxia levels of STAT-3 and the AKT kinase family rose $655 \%$ and 500\%, respectively with the use of propofol anesthesia for the mother.

CONCLUSION: The use of propofol for maternal anesthesia results in less cardiac injury in late-preterm lambs subjected to asphyxia than the use of isoflurane anesthesia. The underlying mechanism may be activation of the antiapoptotic STAT-3 and AKT pathways.

M ulti-organ dysfunction is a common outcome of severe perinatal asphyxia $(1,2)$. One of the organs significantly involved in the fate of the asphyctic newborn is the heart $(3,4)$; it can compromise the oxygen supply of other organs, resulting in irreversible injury (5). It is therefore important to maintain adequate cardiac function in neonates who have undergone an asphyxic insult. Currently, the only available therapy for cardiac function in the neonatal setting after an ischemic insult consists in treatment with positive inotropic and/or vasoactive drugs. The impact of this measure is limited because it neither prevents nor attenuates the degree of the initial myocardial ischemic injury $(6,7)$. Evidence has now accumulated that some anesthetics such as propofol are cardioprotective in adult patients exposed to myocardial ischemia (8-10), and that this propofol-related cardioprotection is mediated through the activation of signal transducer and activator of transcription (STAT)-3 and phosphoinositide-3-kinase/AKT (11). Isoflurane, a volatile anesthetic, also protects the adult heart from ischemia and reperfusion injury by intervening with the mitochondrial apoptosis pathway $(12,13)$. Both anesthetics are rapid acting and reliable and are therefore used when prompt delivery of the fetus becomes essential, as when the fetal heart rate (HR) pattern suggests possible asphyxia or other emergent circumstances necessitating an emergency cesarean section (c-section) (14). Clinical studies in nonemergency c-sections did not show any difference between the two anesthetics with respect to maternal and fetal outcomes (15). However, to our knowledge, there are no studies evaluating risks or benefits of general maternal and fetal anesthesia for the immature heart in preterm infants with severe asphyxia. It is not known whether these two anesthetics modulate the same pathways in the neonatal heart as they do in the adult heart. It is also not clear whether opting for general anesthesia rather than neuraxial anesthesia for c-section in pregnant women carrying a distressed fetus with suspected asphyxia can confer protection for the fetal heart from ischemia and reperfusion injury.

We sought to elucidate these points by establishing an animal model of fetal lambs exposed to severe asphyxia leading to cardiac arrest that are then delivered preterm by an emergency c-section under general anesthesia using propofol or isoflurane (Figure 1). After delivery, the preterm lambs underwent resuscitation followed by $8 \mathrm{~h}$ of intensive care. They received the same sedation regime after birth and resuscitation as their mothers had received during c-section.

\footnotetext{
'Department of Paediatrics, Maastricht University Medical Center, School of Oncology, Maastricht, The Netherlands; ${ }^{2}$ Developmental Biology, Maastricht University Medical Center, School of Mental Health and Neuroscience, Maastricht, The Netherlands; ${ }^{3}$ Department of Pediatric Cardiology, University of Bonn, Bonn, Germany; ${ }^{4}$ Department of Physiology, Cardiovascular Research Institute Maastricht (CARIM), Maastricht University Medical Center, Maastricht, The Netherlands; ${ }^{5}$ Department of Clinical Chemistry, Maastricht University Medical Center, Maastricht, The Netherlands. Correspondence: Boris W. Kramer (B.Kramer@maastrichtuniversity.nl)
} 


\section{Articles | Sechase et al.}

To assess cardiac function in the preterm lambs, left ventricular ejection fraction (LVEF) was determined. This parameter is often depressed after birth asphyxia and is a useful marker of severity and a prognostic indicator in asphyxia (16). In addition, troponin $\mathrm{T}$, a marker of cardiac injury, was measured $(1,17)$. To assess activation or suppression of mitochondrial apoptotic and survival pathways in myocardial left ventricular tissue, the protein level of apoptosis executioner cysteinyl aspartate-specific protease-3 (caspase-3) was determined. Caspase- 3 is activated by caspase- 9 on release of cytochrome $c$ from failing mitochondria in the presence of severe cellular hypoxia (18). B-cell lymphoma-extra large $(\mathrm{Bcl}-\mathrm{xL})$ is a protein capable of mediating cell survival by inhibiting the release of mitochondrial cytochrome $c$ (19). The activity of Bcl-xL is regulated by phosphorylated STAT-3 (pSTAT-3) $(20,21)$ and by AKT $(22)$, both of which were also assessed. The expanding knowledge and about the activation or suppression of molecular death and survival pathways and an improved understanding of their effects and impact on clinical parameters that can be measured at the bedside (such as troponin T and LVEF) may help in creating a therapy that not only influences surrogate end points but also intervenes in the pathogenesis of ischemia and reperfusion injury.

\section{RESULTS}

\section{Physiology}

All the lambs had similar birth weights of $3.6 \pm 0.1 \mathrm{~kg}$. In order to achieve a mean arterial blood pressure (MABP) of $<30 \mathrm{~mm}$ $\mathrm{Hg}$ for $2 \mathrm{~min}$, the total duration of umbilical cord occlusion (UCO) required was $10.7 \pm 0.3 \mathrm{~min}$ in the propofol-treated lambs and $11.3 \pm 0.6 \mathrm{~min}$ in the isoflurane-treated lambs $(P$ $=$ not significant). All the animals developed bradycardia (HR $<30 / \mathrm{min})$ and needed resuscitation. Chest compression was started, and was continued until the HR was $>80 / \mathrm{min}$. The time needed to achieve this HR spontaneously was $9.0 \pm$ $0.3 \mathrm{~min}$ in the propofol-treated lambs and $9.0 \pm 0.4 \mathrm{~min}$ in the isoflurane-treated ones. All the animals that had been exposed to UCO needed adrenaline to recover HR and MABP. The mean dose of adrenaline used was $0.29 \pm 0.10 \mathrm{mg} / \mathrm{kg} \mathrm{BW}$ in the propofol-treated group and $0.32 \pm 0.12 \mathrm{mg} / \mathrm{kgBW}$ in the isoflurane-treated group. HR and MABP values did not differ significantly between the groups after delivery and resuscitation (Figure 2a,b). Initial $\mathrm{pH}$ values before birth were similar in all the groups ( $7.26 \pm 0.01$, Figure 2d). After UCO followed by successful resuscitation, the $\mathrm{pH}$ decreased to $6.91 \pm 0.03$ in the propofol-treated asphyxia group, whereas the propofoltreated control animals (no UCO) showed a pH of 7.10 \pm 0.03 at the corresponding time point after birth. In the isofluranetreated asphyxia group the $\mathrm{pH}$ was $6.84 \pm 0.01$ after resuscitation as compared with $7.07 \pm 0.02$ in the isoflurane-treated control (no UCO) group $(P<0.05)$.

\section{Creatine Kinase in Fetal Plasma}

There were no significant differences among the various groups with respect to changes in creatine kinase (CK) levels (Figure 2c). A trend toward increase was observed in the UCO groups, with CK increasing by $277.1 \%$ in the propofol treatment group and up to $455.7 \%$ in the isoflurane treatment group as compared with the gestational age (GA) control group ( $P=$ not significant).

\section{M-Mode Echocardiography}

Because of logistical limitations, echocardiography could be performed at baseline only in 24 of the 36 late-preterm lambs. Measurements at $8 \mathrm{~h}$ after resuscitation were limited to 16 of the 36 animals. We therefore present the individual measurements per group as compared only with the medians of the groups. Measurements are considered only to determine trends.

Echocardiography at baseline. The median baseline LVEF at $1 \mathrm{~h}$ after birth was $84.0 \%$ in the propofol-treated group and $81.0 \%$ in the isoflurane control group (Figure 3a). The baseline LVEF in asphyctic lambs was $89.0 \%$ in the propofol-treated group and $90.0 \%$ in the isoflurane-treated group. The baseline LVEF was higher after resuscitation and adrenaline treatment in propofol-treated asphyctic lambs than in the corresponding control group. A similar difference was observed between the two isoflurane groups.

Echocardiography after $8 h$. LVEF measurements at $8 \mathrm{~h}$ after birth, immediately before the lambs were killed were $89.5 \%$ and $88.5 \%$ in the propofol and isoflurane control groups,

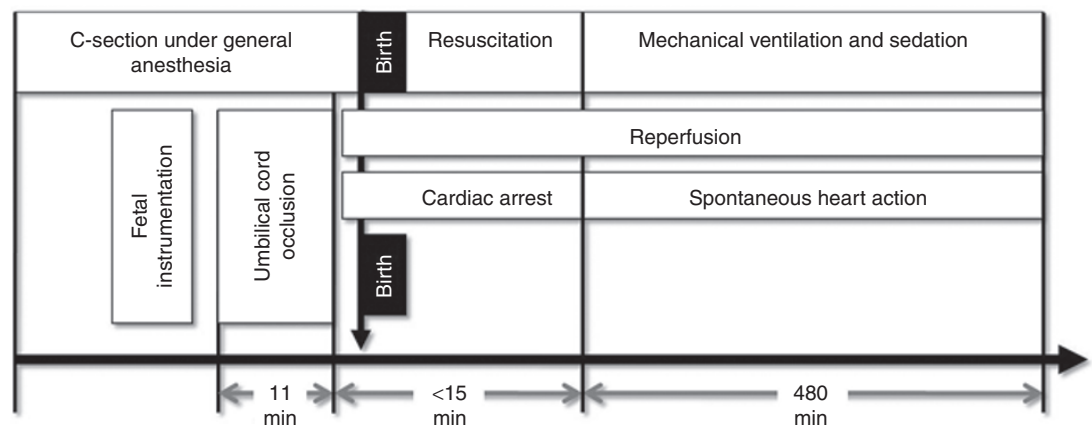

Figure 1. Study design and time course of experiments. The lambs were delivered at $89 \%$ of full gestational age (GA). The ewes carrying the fetuses constituting the GA control group were euthanized, and their offspring were delivered by cesarean section (c-section) and also euthanized. The ewes of the other groups underwent c-section under sedation with the same anesthetic that would subsequently be used for their respective offspring. Two groups of lambs were exposed to total umbilical cord occlusion leading to cardiac arrest. 

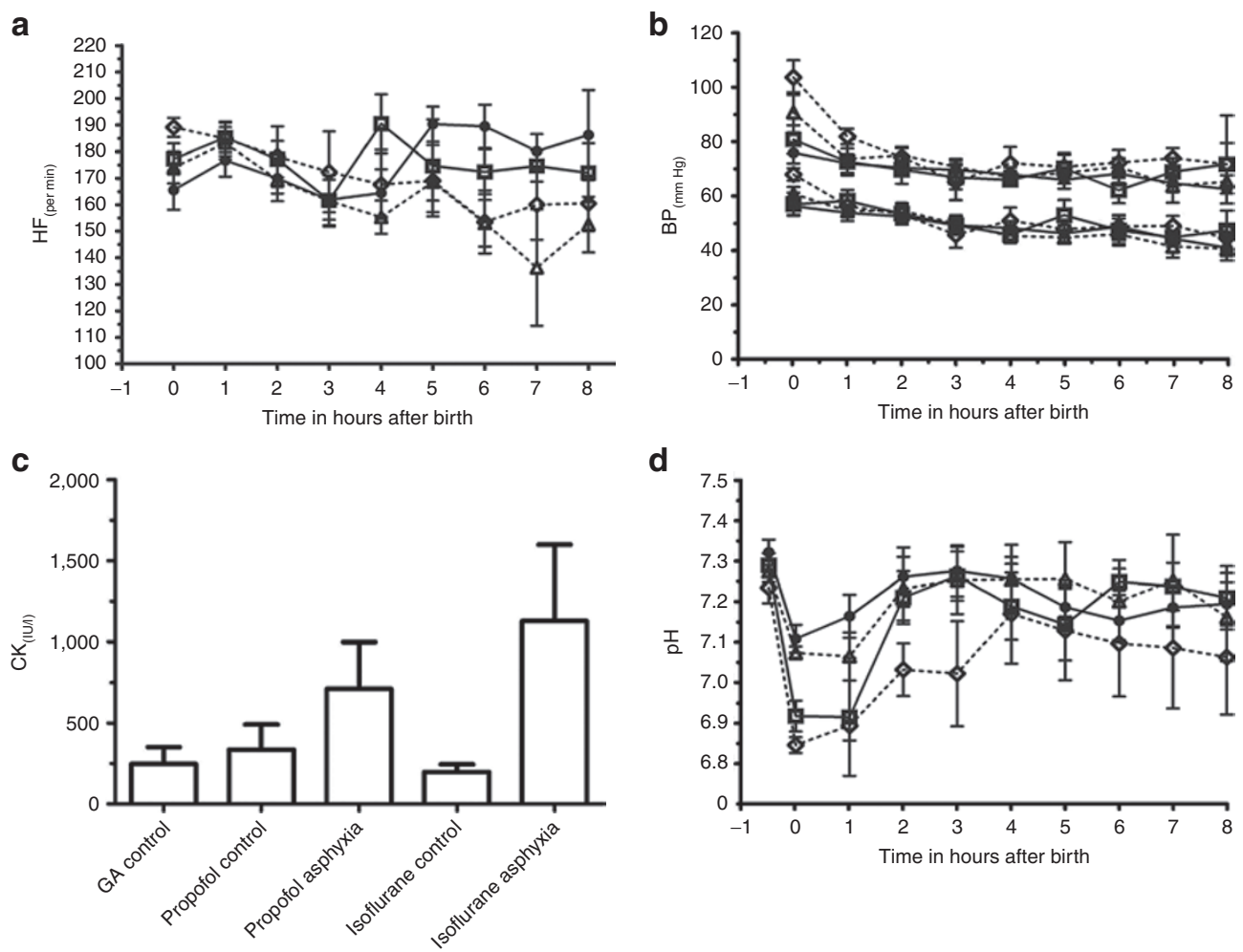

d

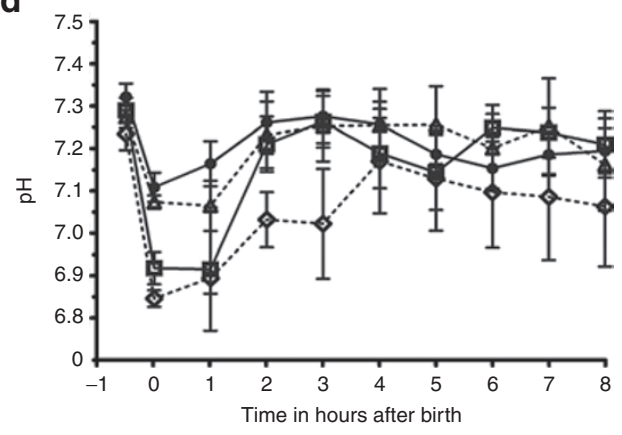

Figure 2. Heart frequency (HF), creatine kinase (CK), blood pressure (BP), and pH in the various groups. (a) HF. (b) Systolic and diastolic blood pressure readings. (c) Creatine kinase in fetal plasma at the end of the experiment. (d) pH values. During the experiments, there were no signs of propofol infusion syndrome, such as bradycardia, arterial hypotension, rhabdomyolysis, or acidosis, in the propofol-treated lambs. The data are depicted as mean values and SEMs. Circles symbolize the propofol control group, squares symbolize the propofol-treated asphyctic group, triangles symbolize the isoflurane control group, and diamonds symbolize the isoflurane-treated asphyctic group. GA, gestational age.

a

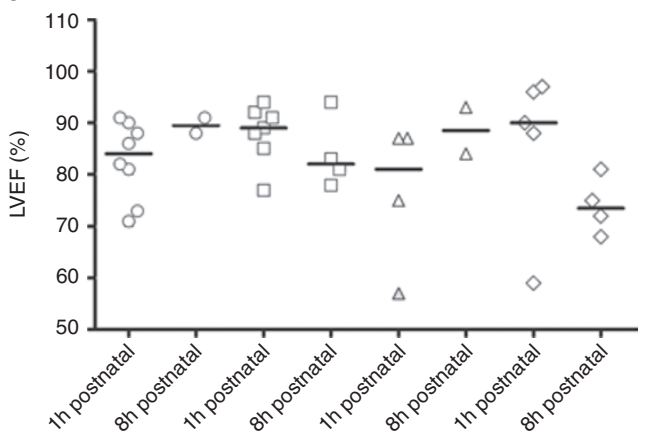

b

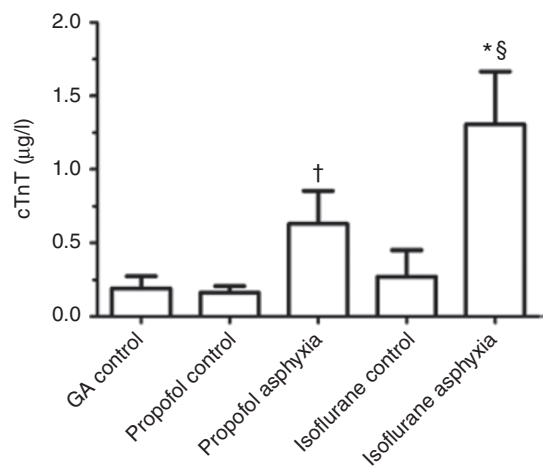

Figure 3. Cardiac parameters left ventricular ejection fraction (LVEF) and cardiac troponin T (cTnT) in various groups. (a) M-mode echocardiography: LVEF. Because of technical reasons, LVEF could not be determined in all the animals. However, a trend for a decreasing median could be observed in the isoflurane-treated asphyctic group at the end of the experiment, whereas the decrease in the propofol-treated asphyctic group was less distinct. Circles symbolize the propofol control group, squares symbolize the propofol-treated asphyctic group, triangles symbolize the isoflurane control group, and diamonds symbolize the isoflurane-treated asphyctic group. (b) cTnT in fetal ovine plasma at the end of the experiment. The data are depicted as mean values and SEMs. Significant differences as compared with gestational age (GA) control groups are $* P<0.05$ (GA group $n=8$, propofol control $n=11$, propofol-treated asphyxia $n=10$; isoflurane control $n=7$, and isoflurane-treated asphyxia $n=8)$. Significant differences $(P<0.05)$ between the propofol control $(n=11)$ and the corresponding isoflurane control group $(n=7)$ are marked by $\S$, and significant differences between the propofol-treated asphyctic group $(n=10)$ and the isoflurane-treated asphyctic group $(n=8)$ are marked by + .

respectively. LVEF values in both propofol-treated and isoflurane-treated animals increased relative to the respective baseline values. The final LVEF in asphyctic lambs was $82.0 \%$ in the propofol treatment group and $73.5 \%$ in the isoflurane treatment group. In comparison with the baseline values,
LVEF decreased in propofol-treated and isoflurane-treated lambs. The LVEF at the end of the experiment was lower in propofol-treated asphyctic lambs than in the propofol-treated control group. A similar difference was observed between the two isoflurane groups. 
a
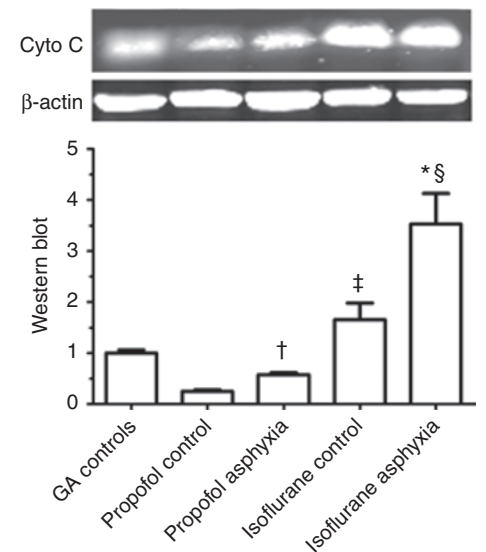

b
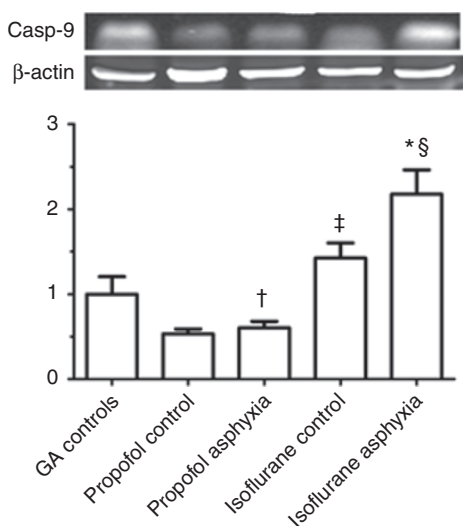

C
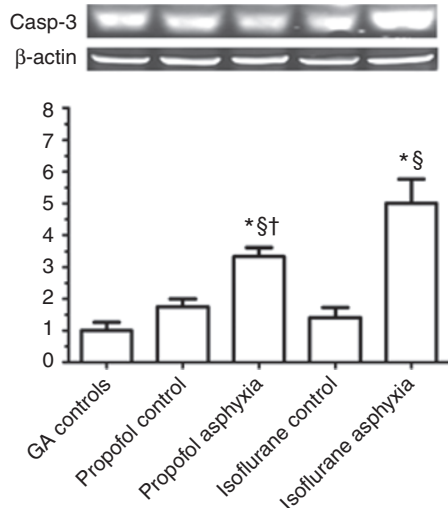

Figure 4. Western blot tests for cytochrome $c$ release into (a) the cytosol, (b) cleaved caspase-9, and (c) caspase-3. The corresponding levels in the gestational age (GA) control group were set at 1. The data are depicted as mean values and SEMs. Significant differences $(P<0.05)$ as compared with GA control groups $(n=8)$ are marked by *. Significant differences $(P<0.05)$ between asphyctic (propofol-treated $n=10$; isoflurane-treated $n=8)$ and the corresponding asphyxia-free control group (propofol-treated $n=11$; isoflurane-treated $n=7$ ) are marked by $\S$. Significant differences between the propofol-treated asphyctic group and the isoflurane-treated asphyctic group are marked by + . Significant differences $(P<0.05)$ between the propofol control group $(n=11)$ and the corresponding isoflurane control group $(n=7)$ are marked by $\neq$.
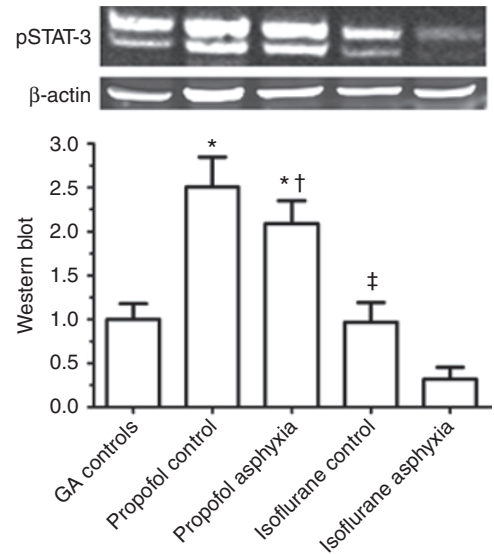

b
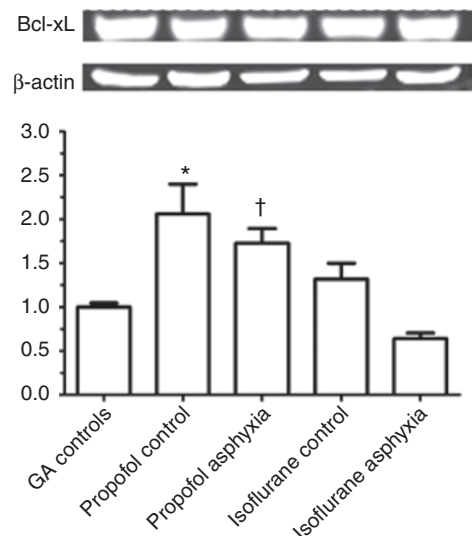

C
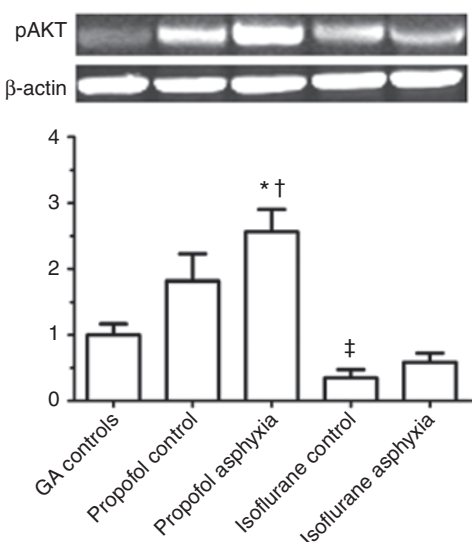

Figure 5. Western blot tests for (a) pSTAT-3, (b) Bcl-xL, and (c) pAKT. The levels of these parameters in the gestational age (GA) control group were set at 1. The data are depicted as mean values and SEMs. Significant differences $(P<0.05)$ as compared with GA control groups are marked by * $(G A$ group $n=8$, propofol control group $n=11$, propofol-treated asphyctic group $n=10$; isoflurane control group $n=7$, isoflurane-treated asphyctic group $n=$ 8). Significant differences $(P<0.05)$ between the propofol control group $(n=11)$ and the corresponding isoflurane control group $(n=7)$ are marked by ‡. Significant differences between the propofol-treated asphyctic group $(n=10)$ and the isoflurane-treated asphyctic group $(n=8)$ are marked by + . PSTAT-3, phosphorylated signal transducer and activator of transcription-3.

\section{Cardiac Troponin T in Fetal Plasma}

In the isoflurane-treated asphyctic group, cardiac troponin $\mathrm{T}$ (cTnT) increased to $1.3 \pm 0.4 \mu \mathrm{g} / \mathrm{l}(P<0.05)$ as compared with the GA control group value of $0.2 \pm 0.1 \mu \mathrm{g} / \mathrm{l}$ (Figure $3 \mathrm{~b}$ ). In the propofol-treated asphyctic group, cTnT increased to $0.6 \pm$ $0.2 \mu \mathrm{g} / \mathrm{l}$ ( $P=$ not significant $)$ as compared with the GA control group. In comparison with isoflurane, propofol reduced cTnT content in fetal plasma after severe asphyxia by $48.2 \%(P<0.05)$.

\section{Cytochrome c-Releasing Apoptosis Assay}

The values of cytochrome $c$ were lower $(57.4 \pm 4.2 \%)$ in the propofol-treated asphyctic group and higher $(166.0 \pm 31.8 \%)$ in the isoflurane-treated asphyctic group relative to the corresponding values in the GA control group ( $P=$ not significant) (Figure 4a).
Cytochrome $c$ values in isoflurane-treated asphyctic lambs were $353.1 \pm 59.5 \%$ of the values in GA controls $(P<0.05), 212.7 \%$ of the values in the isoflurane control group $(P<0.05)$, and $614.8 \%$ of the values in the propofol asphyxia group $(P<0.05)$.

\section{Western Blot Analysis}

Cleaved caspase-9. In the isoflurane-treated asphyxia group, cleaved caspase-9 had increased $217.8 \pm 28.7 \%(P<0.05)$ as compared with GA controls and $360.4 \%(P<0.05)$ as compared with the propofol-treated asphyxia group (Figure $4 \mathrm{~b}$ ).

Cleaved caspase-3. In the propofol-treated asphyxia group, cleaved caspase-3 had increased $333.5 \pm 27.8 \%(P<0.05$, Figure 4c) as compared with the GA controls, and 190.2\% ( $P$ 


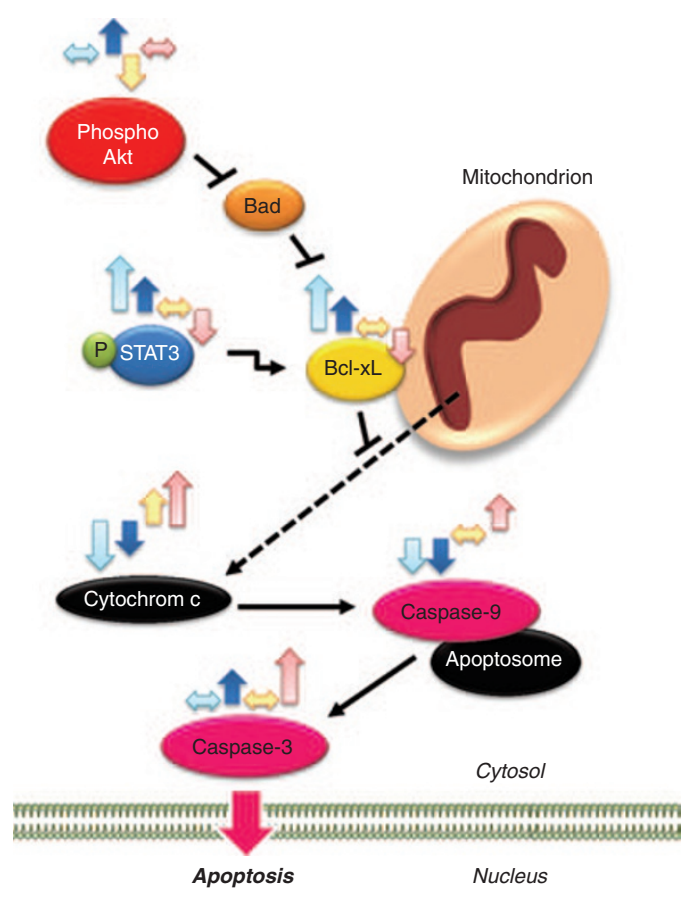

Figure 6. Activation and inhibition of apoptotic pathways in the fetal ovine heart. Propofol treatment resulted in activation of PSTAT- 3 and pAkt, which inhibited release of cytochrome $c$ from the mitochondria. Consequently, activation of caspases- 9 and -3 was reduced. Colored arrows symbolize increase (upward arrow), decrease (downward arrow), or no significant change (left-right arrow) in the levels of the analyzed target protein. The different experimental groups are depicted in the following colors: light blue (propofol control), dark blue (propofol-treated asphyctic), yellow (isoflurane control), and red (isoflurane-treated asphyctic). A straight black arrow means "activation," a straight black arrow with a horizontal line instead of an arrow head means "inhibition," an arrow with a broken line means "release," and an arrow with a turning shaft means "transcriptional stimulation" of the respective target protein. Bcl-xL, B-cell lymphoma-extra large; pAKT, phosphorylated AKT; PSTAT-3, phosphorylated signal transducer and activator of transcription-3.

$<0.05)$ as compared with the propofol-treated control group. Values of cleaved caspase- 3 in isoflurane-treated asphyctic lambs increased $500.5 \pm 76.2 \%$ as compared with GA controls $(P<0.05), 355.5 \%(P<0.05)$ as compared with the isoflurane control group, and $150.1 \%(P<0.05)$ as compared with the propofol-treated asphyctic group.

pSTAT-3 and Tyr705. The protein level of pSTAT-3 increased $250.5 \pm 34.1 \%$ in the propofol control group as compared with the GA control group $(P<0.05$; Figure $5 \mathrm{a})$. In the propofol-treated asphyctic group, pSTAT-3 was higher by $209.2 \pm 25.7 \%(P<0.05)$ as compared with the GA control group and by $655.8 \%(P<0.05)$ as compared with the isoflurane-treated asphyctic group. The pSTAT-3 value in the isoflurane control group was similar to that in the GA control group, whereas in the isoflurane-treated asphyctic group it was lower $(31.9 \pm 13.4 \%)$ as compared with the GA controls $(P=$ not significant $)$.

$B c l-x L$. The protein level of Bcl-xL was higher $(206.1 \pm 33.9 \%)$ in the propofol control group as compared with the GA control group $(P<0.05$; Figure $5 \mathrm{~b})$. The $\mathrm{Bcl}-\mathrm{xL}$ value in the propofol-treated asphyctic group was higher $(172.6 \pm 17.1 \%)(P=$ not significant) as compared with the GA control group and higher $(268.8 \%)(P<0.05)$ as compared with the isoflurane asphyxia group as well. There was no difference in Bcl-xL values between the isoflurane control group and the GA control group. The Bcl-xL value in the isoflurane-treated asphyctic group was lower $(64.2 \pm 6.4 \%)$ as compared with GA controls $(P=$ not significant $)$ and higher $(205.1 \%)(P=$ not significant $)$ as compared with the isoflurane control group.

Phosphorylated AKT. The protein level of pAKT in the propofol-treated asphyctic group was higher $(256.3 \pm 34.0 \%)$ as compared with the GA control group $(P<0.05)$ (Figure $5 c)$. The pAKT value in the isoflurane-treated asphyctic group was lower $(22.9 \%)$ as compared with the propofol-treated asphyctic group $(P<0.05)$.

\section{DISCUSSION}

In this article, we demonstrated, for the first time, functional and molecular changes that take place in the hearts of latepreterm lambs. We used a unique experimental sequence of severe asphyxia brought about by complete UCO in utero, fetal cardiac arrest, emergency c-section, postnatal resuscitation of the fetus, and intensive care for the first $8 \mathrm{~h}$ after birth. The molecular and functional changes indicated that the choice of sedative drugs has the potential to mediate cardioprotection in the asphyctic fetus when administered before the actual delivery to the maternal-fetal unit during c-section, and postnatally to the survivors in the neonatal intensive care unit.

Cardiac dysfunction is a common feature in asphyxiated preterm infants, but is often underdiagnosed; it therefore requires a high index of suspicion $(3,4)$. Echocardiography helps in early recognition and better management of these cases. LVEF is a useful marker of severity and prognosis of cardiac dysfunction related to perinatal asphyxia (16). We were able to show, for the first time, that the choice of anesthesia for emergency c-section in the mother can influence the heart function of a lamb fetus that has undergone severe asphyxia. Abboud et al. showed that, in the absence of fetal asphyxia, neonatal status was equally good regardless of whether the anesthetic used during the c-section was propofol or isoflurane (15). This observation was congruent with the findings in our control groups of late-preterm lambs after the use of either isoflurane or propofol as sedation in the ewes. The choice of anesthetic for the pregnant animal during c-section had an impact on neonatal status only if the fetus had received exposure to severe asphyxia followed by resuscitation. In late-preterm asphyctic lambs, LVEF was re-established better after propofol treatment than after isoflurane treatment. Echocardiography showed that anesthesia with isoflurane was associated with a trend toward higher levels of cTnT in the fetal plasma of severely asphyctic lambs than anesthesia with propofol. TnT is a marker of cardiac damage in preterm and term-born infants and is a strong indicator of myocardial injury caused by perinatal hypoxia $(1,17)$. Therefore, it can be speculated that the absence of an 


\section{Articles | seehase etal.}

increase in cTnT when propofol treatment is used may be a sign of cardioprotection.

The lower rate of release of cTnT into fetal plasma and the improvement in heart function in asphyctic late-preterm lambs treated with propofol may be partially attributable to a reduced rate of apoptosis. In our study, activation of myocardial caspase-3, which determines the incidence of apoptosis (19), was significantly reduced by propofol treatment. Wang et al. provided evidence that propofol might interfere with apoptotic pathways through activation of AKT (11). Activated AKT blocks apoptosis by phosphorylating its substrates, such as $\mathrm{Bcl}-2$-associated death protein (Bad) (23). The latter, in turn, modulates Bcl-xL, an antiapoptotic protein (19). In support of this hypothesis, we found that propofol-treated groups showed increased cardiac protein levels of phosphorylated $\mathrm{AKT}$ and $\mathrm{Bcl}-\mathrm{xL}$ as well as slightly better LVEF values as compared with the isoflurane-treated groups. Indeed, isoflurane caused no change in cardiac pAKT and Bcl-xL. The increase in $\mathrm{pAKT}$ and $\mathrm{Bcl}-\mathrm{xL}$ after fetal exposure exposure to propofol is thought to lead to lower levels of cytochrome $c$ being released from the mitochondria, and consequently to less activation of caspase- 9 and caspase- 3 in fetal hearts. Some previous studies have demonstrated that the level of phosphorylated AKT may reflect its activity under conditions such as preconditioning (24) and postconditioning (23). Therefore, the findings of our study suggest that propofol administration to the mother and to the fetus at the onset of reperfusion for $8 \mathrm{~h}$ might attenuate myocardial apoptosis, partly through maintaining or even increasing the activity of the phosphoinositide-3-kinase/AKT pathway.

Another pathway that contributes to myocardial protection in ischemic postconditioning is activation of pSTAT-3 $(25,26)$. A recent study by Bolli et al. has provided evidence that recruitment of STAT3 plays an essential role in the upregulation of cardioprotective and antiapoptotic proteins; the evidence further suggests that STAT3 activation is important in inhibiting both the death receptor pathway and the mitochondrial pathway (27). STAT-3 regulates the transcription of Bcl-xL $(20,21)$. It is currently not known whether this pathway can be activated by propofol. In view of the fact that myocardial protection by ischemic postconditioning and by anesthetic drugs seem to share many pathways, we determined the protein levels of pSTAT-3 in the fetal hearts. We found that pSTAT-3 was indeed increased by propofol but not by isoflurane. Our data, combined with those in the cited literature, indicate that propofol may exert a cardioprotective effect by activation of the STAT-3 and AKT pathways, leading to activation of Bcl-xL. The consequent reduction in cytochrome $c$ release from the mitochondria would have the effect of reducing caspase- 9 and -3 activation (Figure 6).

Our study has several limitations. First, the control group also received a sedative drug. In order to capture the true effect of propofol on cardiac function, one would have to compare against an untreated control group. However, from the clinical and ethical points of view, it was not feasible to maintain late-preterm lambs without sedation and ventilation after subjecting them to asphyxia and subsequent resuscitation. Therefore, for the purpose of controls, we evaluated lambs that had not been subjected to asphyxia.

Second, although the levels of cTnT and the molecular mediators were measured in all the animals at all time points, the assessment of LVEF was not performed in all the animals at all time points because of logistical constraints. Therefore, the relevance of the echocardiographic results is limited. However, the trends observed for LVEF corresponded to the data obtained in cTnT assay and western blot, and we therefore incorporated the echocardiographic data into the manuscript despite their limited relevance.

Third, a risk associated with the postnatal use of propofol in neonates is the possible occurrence of propofol infusion syndrome (28). This is characterized by hemodynamic abnormalities, lactic acidosis, and rhabdomyolysis, and is associated with a mortality of $\sim 85 \%$ (3). The propofol-treated lambs in our study developed no acidosis, no increase in $\mathrm{CK}$, and no severe hemodynamic disturbances within $8 \mathrm{~h}$ (Figure 2). But we cannot rule out the possibility that the use of propofol for a longer duration or in a bigger sample of individuals could lead to propofol infusion syndrome, given the relatively high interindividual variability in propofol pharmacokinetics in preterm and term neonates (5). On the other hand, the human fetus is not affected by propofol anesthesia to the mother during c-section (15). Therefore, future studies should evaluate whether the use of propofol in human fetuses in the prenatal stage alone (and not in the postnatal situation) would be effective in reducing fetal cardiac injury caused by severe asphyxia.

In this study, we were able to show in our animal model of late-preterm sheep that, in the absence of asphyxia, there is no difference in the cardiac status of the newborn animals, irrespective of the anesthetic used (propofol or isoflurane). This has already been shown in human newborns (15). Our study provided evidence, for the first time, that propofol treatment, as compared with isoflurane treatment, results in less myocardial injury and apoptosis and in a better cardiac output in late-preterm lambs after exposure to severe asphyxia and resuscitation. To our knowledge, this is the first report to demonstrate a clinical benefit for the fetus from the experimental concept of ischemic pre- and postconditioning. The fact that anesthetic drugs such as propofol can be administered easily to the fetus through placental perfusion even before birth offers a new and very early therapeutic option for either pharmacological preconditioning of a fetus at risk or for postconditioning of a fetus that has already been exposed to asphyxia. In this context, our study is the first step toward re-evaluating the therapeutic option of using propofol for maintaining and modulating the cardiac function of the fetus during and after perinatal asphyxia. The possibility of pharmacological treatment of the asphyctic fetus through its mother offers new opportunities for therapeutic strategies in the delivery room. These require intensive cooperation between anesthesiologist, obstetrician, and pediatrician. More studies are needed in order to evaluate whether our findings of clinical benefit are limited to the animal model, or whether human newborns too may profit from early treatment in utero. 
In summary, we were able to show that propofol treatment prenatally and in the first hours of life may help to preserve LVEF in late-preterm lambs exposed to severe asphyxia and cardiac arrest, and that this intervention reduces the release of cTnT into fetal plasma. This beneficial effect seems to be mediated, at least in part, by an induction of the STAT-3 and AKT pathway, resulting in reduced release of cytochrome $c$ from the mitochondria and less activation of apoptosis executioner caspase-3. These results could provide the rationale for a clinical study to evaluate the effects of propofol anesthesia in the delivery room for mothers who are carrying fetuses at risk for asphyxia.

\section{METHODS}

Animals

The study was approved by the Animal Ethics Research Committee, Maastricht University, The Netherlands. Texel ewes were date-mated, and the fetuses were randomized for UCO and sedation. Observations were made in 44 late-preterm fetal sheep of both genders at a GA of 133 or $134 \mathrm{~d}$ (term $150 \mathrm{~d}$ ). Eighteen sheep fetuses were subjected to total UCO in utero, and 18 fetal sheep served as sham controls. In each group, half of the pregnant ewes and their offspring were sedated with propofol and the others with isoflurane. Eight lambs served as GA controls for reference values for the molecular pathways in this age group. In order to avoid any influence of anesthetic drugs in the latter group, the pregnant ewes were euthanized using an injection of T61 (Veterinaria AG, Zürich, Switzerland) at gestational day 133 or 134; their offspring were delivered surgically immediately thereafter and were also directly euthanized using an injection of T61 (Veterinaria AG).

\section{Experimental Protocol}

The pregnant ewes of the isoflurane group were intubated after thiopenthal induction $(15 \mathrm{mg} / \mathrm{kg})$, and general anesthesia was maintained with isoflurane $(1-2 \%)$. The pregnant ewes of the propofol group received propofol both as induction bolus $(6 \mathrm{mg} / \mathrm{kg})$ and for general anesthesia $(25 \mathrm{mg} / \mathrm{kg} / \mathrm{h})$ during the c-section. In both groups of animals, anesthesia was supplemented by continuous remifentanyl infusion $(3 \mu \mathrm{g} / \mathrm{kg} / \mathrm{min})$. After a lower-midline incision, the fetus was carefully extracted through a small incision in the uterus. An endotracheal tube was inserted into the fetus and clamped to prevent breathing. Catheters were placed in the femoral and umbilical arteries and in the jugular vein for baseline blood sampling (Abbott i-STAT 1 Blood Gas Analyzer; Abbott Laboratories, Abbott Park, IL) and for continuous monitoring of fetal MABP and HR. Electrocardiogram and electroencephalogram electrodes were put in place and stitched to the skin. The umbilical cord was gently extracted through an incision in the uterus, and a vascular occluder (OC16HD, $16 \mathrm{~mm}$; In Vivo Metric, Healdsburg, CA) was placed around the umbilical cord. The aim of the occlusion was to achieve a MABP of $<30 \mathrm{~mm} \mathrm{Hg}$ for $2 \mathrm{~min}$ (Figure 1). At the end of the occlusion period, the lambs had experienced severe bradycardia $(\mathrm{HR}<$ $30 / \mathrm{min}$ ) or even complete cardiac arrest. If there was no increase in HR at $20 \mathrm{sec}$ after the end of the occlusion period, resuscitation was started. The resuscitation protocol included immediate start of cardiac massage at a frequency of $120 / \mathrm{min}$ and adrenaline administration in augmented doses from $30 \mu \mathrm{g}$, to $60 \mu \mathrm{g}$, to $0.1 \mathrm{mg}$. In addition, a volume bolus of ringer lactate of $10 \mathrm{ml} / \mathrm{kg} \mathrm{BW}$ was administered after the first shot of adrenaline, and ventilation was administered using a balloon $\left(\mathrm{FiO}_{2}\right.$ $=0.21$, frequency $=60 / \mathrm{min}$ ). After cutting of the umbilical cord, the fetus was brought to an open, heated incubator (IW930 Series CosyCot Infant Warmer; Fisher \& Paykel Healthcare, Auckland, New Zealand), and body temperature was maintained at $38.5^{\circ} \mathrm{C}$ while resuscitation was continued. The lambs were administered pressure-regulated ventilation using a ventilator Servo 900C (Siemens-Elema, Solna, Sweden) with initial settings as follows: $\mathrm{FiO}_{2}=1$, positive end-expiratory pressure, $5 \mathrm{cmH}_{2} 0$, peak inspiratory pressure $30 \mathrm{cmH}_{2} 0$, frequency $60 / \mathrm{min}$, I:E 1:2. Thereafter, inspiratory pressure was adjusted to achieve a targeted volume of $3.0 \mathrm{~L} / \mathrm{min}$ and a $\mathrm{P}_{\mathrm{a}} \mathrm{CO}_{2}$ of $35-45 \mathrm{~mm} \mathrm{Hg}$. Ventilation and sedation were continued for $8 \mathrm{~h}$ after the delivery. Sedation was maintained either with isoflurane (0.5-1.0\%) or with propofol (1-3 mg/ $\mathrm{kg} / \mathrm{h})$, supplemented with remifentanyl $(3 \mu \mathrm{g} / \mathrm{kg} / \mathrm{min})$ in both groups. The remifentanyl was dissolved in $20 \%$ glucose and ringerlactate solution in a concentration allowing for infusion at the rate of $3 \mathrm{ml} / \mathrm{kg} / \mathrm{h}$. All the lambs (sham treated included) received surfactant $(200 \mathrm{mg} / \mathrm{kg}$ BW Curosurf, courtesy of Chiesi, Parma, Italy) intratracheally, and two times maternal blood transfusion of $10 \mathrm{ml} / \mathrm{kg}$ BW during initial stabilization. All cord-occluded lambs except two spontaneously developed a $\mathrm{HR}>150 / \mathrm{min}$ and sufficient MABP $(>50 \mathrm{~mm} \mathrm{Hg})$ within $10 \mathrm{~min}$ after the start of resuscitation; the two lambs were excluded from the study.

The 18 sham-treated fetuses were prepared in utero, with insertion of catheters in the femoral and umbilical arteries and jugular vein and endotracheal intubation, as described above. At 11 min (mean duration of occlusion in the study group) after the end of the instrumentation procedure, the lambs were delivered, weighed, sedated, and connected to pressure-controlled ventilation as described above.

At $8 \mathrm{~h}$ after delivery, the lambs were euthanized using an intravenous injection of T61 (Veterinaria AG). The heart was immediately removed and shock-frozen in liquid nitrogen. Tissues were subsequently stored at $-80^{\circ} \mathrm{C}$.

\section{M-Mode Echocardiography}

Transthoracic echocardiography using a 5.2-MHz transducer (Megas CVX, Esaote, Genova, Italy) was performed at baseline (1 $\mathrm{h}$ after birth) and prior to euthanasia ( $8 \mathrm{~h}$ after birth). M-mode recordings from a parasternal short-axis view were used to determine LV end-diastolic diameter and LV end-systolic diameter in accordance with the recommendations of the American Society of Echocardiography. The investigator performing and reading the echocardiogram was blinded to the treatment allocation.

\section{Western Blot Test}

Frozen left ventricular heart tissue was homogenized in ice-cold radioimmunoprecipitation assay buffer (Sigma-Aldrich, St. Louis, MO) containing Halt Protease and Phosphatase Inhibitor Cocktail, EDTA-free (100X) (Thermo Fisher Scientific, Rockford, IL). The samples were then centrifuged at $500 \mathrm{~g}$ for $20 \mathrm{~min}$ at $4^{\circ} \mathrm{C}$ to remove cellular debris. Protein content in the supernatant was determined using the Micro BCA Protein Assay Kit (Thermo Fisher Scientific), with bovine serum albumin as the standard. Western blots were performed as described previously (29). The following dilutions were used for antibodies: 1:1,000 for monoclonal anti- $\beta$-actin clone AC-1 (Sigma-Aldrich), anticleaved-caspase-3 (Asp175) (Cell Signaling Technology, Danvers, MA) (30), anticleavedcaspase-9 (Novus Biologicals, Littleton, CO), anti-Bcl-xL (Cell Signaling Technology), anti-phospho-AKT (Cell Signaling Technology), and antipSTAT-3 (Cell Signaling Technology). As secondary antibody, IRDye 680 conjugated goat (polyclonal) antirabbit IgG, IRDye 800 conjugated goat (polyclonal) antirabbit IgG, IRDye 680 conjugated goat (polyclonal) antimouse IgG, and IRDye 800 conjugated goat (polyclonal) antimouse IgG, (LI-COR, Lincoln, NE) were used in dilutions of 1:10,000. The blots were analyzed using the LICOR Odyssey Infrared Imaging System, and the signals of the target proteins were normalized to the signal of $\beta$-actin, which acts as a housekeeping protein. Images were acquired using Adobe Photoshop CS4 software (San Jose, CA).

\section{Cytochrome c-Releasing Apoptosis Assay Kit}

The cytochrome $c$ release rate was determined using the cytochrome $c$-releasing apoptosis assay kit (BioVision, Mountain View, CA) in accordance with the manufacturer's protocol.

\section{Measurement of Blood Biochemical Variables}

Troponin T (fourth-generation TnT assay) content in fetal ovine plasma was analyzed on the Elecsys 2010 (Roche Diagnostics, Basel, Switzerland).

CK plasma concentration was determined on a Beckman Coulter LX20 PRO Clinical Chemistry analyzer (Beckman Coulter, Fullerton, CA). The analysis was performed as described in the manual (https:// www.beckmancoulter.com/wsrportal/page/techdocSearch).

\section{Statistical Analysis}

Measurements of LVEF were depicted as median values. All other data were depicted as mean \pm SEM. The statistical significance of 
intergroup differences was analyzed using ANOVA followed by the Student-Newman-Keuls multiple-comparison test. Differences with probability values of $P<0.05$ were considered significant. All statistical analyses were performed using the statistical software GraphPad Prism 5.0 (La Jolla, CA).

\section{ACKNOWLEDGMENTS}

We thank Monique Engel for her advice and for making the technical equipment available to us. We also thank Claudia Lorefice, Elke Kuypers, Lotte van den Heuij, and the staff of the central animal facility of Maastricht University for their excellent technical help.

\section{STATEMENT OF FINANCIAL SUPPORT}

This work was supported by the Foundation of Pediatrics (Stichting Kindergeneeskunde), Maastricht University Medical Center.

Disclosures: The authors declared no conflict of interest.

\section{REFERENCES}

1. Szymankiewicz M, Matuszczak-Wleklak M, Vidyasagar D, Gadzinowski J. Retrospective diagnosis of hypoxic myocardial injury in premature newborns. J Perinat Med 2006;34:220-5.

2. Rennie JM, Hagmann CF, Robertson NJ. Outcome after intrapartum hypoxic ischaemia at term. Semin Fetal Neonatal Med 2007;12:398-407.

3. Shah P, Riphagen S, Beyene J, Perlman M. Multiorgan dysfunction in infants with post-asphyxial hypoxic-ischaemic encephalopathy. Arch Dis Child Fetal Neonatal Ed 2004;89:F152-5.

4. Hankins GD, Koen S, Gei AF, Lopez SM, Van Hook JW, Anderson GD. Neonatal organ system injury in acute birth asphyxia sufficient to result in neonatal encephalopathy. Obstet Gynecol 2002;99(5 Pt 1):688-91.

5. Kanik E, Ozer EA, Bakiler AR, et al. Assessment of myocardial dysfunction in neonates with hypoxic-ischemic encephalopathy: is it a significant predictor of mortality? J Matern Fetal Neonatal Med 2009;22:239-42.

6. Pellicer A, Valverde E, Elorza MD, et al. Cardiovascular support for low birth weight infants and cerebral hemodynamics: a randomized, blinded, clinical trial. Pediatrics 2005;115:1501-12.

7. Obaid L, Johnson ST, Emara M, Bigam DL, Cheung PY. Epinephrine versus dopamine to treat shock in hypoxic newborn pigs resuscitated with $100 \%$ oxygen. Shock 2008;29:262-8.

8. Bovill JG. Intravenous anesthesia for the patient with left ventricular dysfunction. Semin Cardiothorac Vasc Anesth 2006;10:43-8.

9. Vasileiou I, Xanthos T, Koudouna E, et al. Propofol: a review of its nonanaesthetic effects. Eur J Pharmacol 2009;605:1-8.

10. Yildirim V, Doganci S, Aydin A, Bolcal C, Demirkilic U, Cosar A. Cardioprotective effects of sevoflurane, isoflurane, and propofol in coronary surgery patients: a randomized controlled study. Heart Surg Forum 2009;12:E1-9.

11. Wang B, Shravah J, Luo H, Raedschelders K, Chen DD, Ansley DM. Propofol protects against hydrogen peroxide-induced injury in cardiac H9c2 cells via Akt activation and Bcl-2 up-regulation. Biochem Biophys Res Commun 2009;389:105-11.

12. Ge ZD, Pravdic D, Bienengraeber M, et al. Isoflurane postconditioning protects against reperfusion injury by preventing mitochondrial perme- ability transition by an endothelial nitric oxide synthase-dependent mechanism. Anesthesiology 2010;112:73-85.

13. Kalenka A, Maurer MH, Feldmann RE, Kuschinsky W, Waschke KF. Volatile anesthetics evoke prolonged changes in the proteome of the left ventricule myocardium: defining a molecular basis of cardioprotection? Acta Anaesthesiol Scand 2006;50:414-27.

14. Rollins M, Lucero J. Overview of anesthetic considerations for Cesarean delivery. Br Med Bull 2012;101:105-25.

15. Abboud TK, Zhu J, Richardson M, Peres Da Silva E, Donovan M. Intravenous propofol vs thiamylal-isoflurane for caesarean section, comparative maternal and neonatal effects. Acta Anaesthesiol Scand 1995;39: 205-9.

16. Ranjit MS. Cardiac abnormalities in birth asphyxia. Indian J Pediatr 2000;67:529-32.

17. Cruz MA, Bremmer YA, Porter BO, Gullquist SD, Watterberg KL, Rozycki HJ. Cardiac troponin T and cardiac dysfunction in extremely low-birthweight infants. Pediatr Cardiol 2006;27:396-401.

18. Jensen FE. The role of glutamate receptor maturation in perinatal seizures and brain injury. Int J Dev Neurosci 2002;20:339-47.

19. Reed JC. Mechanisms of apoptosis. Am J Pathol 2000;157:1415-30.

20. Grad JM, Zeng XR, Boise LH. Regulation of Bcl-xL: a little bit of this and a little bit of STAT. Curr Opin Oncol 2000;12:543-9.

21. Zhuang L, Lee CS, Scolyer RA, et al. Mcl-1, Bcl-XL and Stat3 expression are associated with progression of melanoma whereas Bcl-2, AP-2 and MITF levels decrease during progression of melanoma. Mod Pathol 2007;20:416-26.

22. Rees S, Harding R, Walker D. The biological basis of injury and neuroprotection in the fetal and neonatal brain. Int $J$ Dev Neurosci 2011;29:551-63.

23. Eigel BN, Gursahani H, Hadley RW. ROS are required for rapid reactivation of $\mathrm{Na}+/ \mathrm{Ca} 2+$ exchanger in hypoxic reoxygenated guinea pig ventricular myocytes. Am J Physiol Heart Circ Physiol 2004;286:H955-63.

24. Armstrong SC. Protein kinase activation and myocardial ischemia/reperfusion injury. Cardiovasc Res 2004;61:427-36.

25. Holopainen IE, Laurén HB. Glutamate signaling in the pathophysiology and therapy of prenatal insults. Pharmacol Biochem Behav 2012;100:825-34.

26. Eigel BN, Gursahani H, Hadley RW. Na+/Ca2+ exchanger plays a key role in inducing apoptosis after hypoxia in cultured guinea pig ventricular myocytes. Am J Physiol Heart Circ Physiol 2004;287:H1466-75.

27. Bolli R, Stein AB, Guo Y, et al. A murine model of inducible, cardiacspecific deletion of STAT3: its use to determine the role of STAT3 in the upregulation of cardioprotective proteins by ischemic preconditioning. J Mol Cell Cardiol 2011;50:589-97.

28. Bray RJ. Propofol infusion syndrome in children. Paediatr Anaesth 1998;8:491-9.

29. Seehase M, Gantert M, Ladenburger A, et al. Myocardial response in preterm fetal sheep exposed to systemic endotoxinaemia. Pediatr Res 2011;70:242-6.

30. Kunzmann S, Glogger K, Been JV, et al. Thymic changes after chorioamnionitis induced by intraamniotic lipopolysaccharide in fetal sheep. Am J Obstet Gynecol 2010;202:476.e1-9. 